\title{
Influence of electron distribution on efficiency droop for GaN-based light emitting diodes
}

\author{
Jiajia Fu ${ }^{1,2}$, Lixia Zhao ${ }^{1,2^{*}}$, Ning Zhang ${ }^{1,2}$, Junxi Wang ${ }^{1,2}$ and Jinmin $\mathrm{Li}^{1,2}$
}

\footnotetext{
* Correspondence: Ixzhao@semi.ac.cn ${ }^{1}$ Semiconductor Lighting Research and Development Center, Institute of Semiconductors, Chinese Academy of Sciences, 100083 Beijing, People's Republic of China

${ }^{2}$ State Key Laboratory of Solid-State Lighting, Institute of Semiconductors, Chinese Academy of Sciences, 10083, Beijing, People's Republic of China
}

\begin{abstract}
By modulating the indium composition in the quantum barriers of InGaN-based LEDs, the influence of electron distribution, electron overflow and Auger recombination on the external quantum efficiency (EQE) and droop effect have been investigated. Experimental results as well as numerical simulations reveal that the electron distribution is the key factor to influence both the peak efficiency and droop effect. The results show that the high electron concentration in the individual quantum well can stimulate the Auger recombination and lead to the droop effect instead of the total effective electron concentration, which is more related to the external quantum efficiency. If we modulate the indium composition in the quantum barriers of the InGaN-based LEDs, a uniform electron distribution can be achieved, which can not only enhance the EQE but also avoid the Auger recombination and improve the droop effect.

Keywords: InGaN quantum barriers; Electron distribution; Carrier recombination; Efficiency droop
\end{abstract}

\section{Background}

GaN-based light-emitting diodes have been developed significantly in recent years and have already been used in many applications, such as general illumination, full color display, back lighting and communications. But for these applications, LEDs need to be operated at a current density below $10 \mathrm{~A} / \mathrm{cm}^{2}$ because of droop effect, which is still a key problem obstructing the development of high-power lighting-emitting diodes. Till now, lots of mechanisms have already been suggested to explain the "efficiency droop", such as polarization effect [1, 2], electron leakage out of the active region [3, 4], carrier delocalization [5], carrier injection efficiency [6], Auger recombination [7] and defects [5]. But the physical mechanisms of the droop effect aren't entirely clear and there is still a debate between them. In this study, we try to understand the relationship between these suggested mechanisms behind the droop effect and our findings will pave a way to further increase the EQE of LEDs at high current density.

Efficiency droop is mainly related to the non-radiative carrier loss mechanism at high injection current. This non-radiative recombination can occur either in the active region's QWs or in the p-type region. In general, non-radiative recombinations, including defect-related Shockley-Read-Hall (SRH) recombination and Auger recombination, 
are more concentrated in the QWs, whereas electron overflow resulting in nonradiative recombination is in the p-type region [8].

The conventional $\mathrm{ABC}$ model is presented as:

$$
I Q E=B n^{2} /\left(A n+B n^{2}+C n^{3}\right),
$$

which can explain the carrier recombination process to a certain extent, where A, B, C represent the Shockley-Read-Hall (SRH), radiative and Auger coefficients, respectively, and $\mathrm{n}$ is carrier concentration [9-11]. Under a large current injection condition, electron concentration is approximate to hole concentration in the active region, and carrier concentration represents electron concentration or hole concentration [12]. Based on this $A B C$ model, IQE-versus-n curve should be symmetry regarding to the peak IQE, which is different from the experimental asymmetry corresponding curve. Given the electron overflow, the $\mathrm{ABC}$ model can be written as follows:

$$
I Q E=B n^{2} /\left[A n+B n^{2}+C n^{3}+f(n)\right],
$$

where the high power term $f(n)$ is the electron overflow term $[13,14]$. If taking the carrier localization into account as well, the effective volume of the active region will be introduced [15, 16], and compared with the conventional $\mathrm{ABC}$ model, the corrected $\mathrm{ABC}$ model will exhibit better fitting to the experimental results. However, in these analytic processes, it has been assumed that carrier concentration in each quantum well is equal and did not consider different levels of the electron leakage in the samples.

Here, we modulated the indium concentration in the quantum barriers of InGaN/GaN MQWs without electron blocking layer and investigated the carrier distribution in quantum well region as well as its influence on the efficiency droop. The replacement of the $\mathrm{GaN}$ barriers by InGaN will not only reduce the polarization effect between the quantum barriers and quantum wells [17], but change the conductor band offset $\Delta E_{c}$, which will result in different electron overflow and carrier distribution and allow us to investigate the influence on the efficiency droop. We found that actually the electron distribution is the key factor, which will influence the electron overflow and Auger recombination and affect the droop effect together (Fig. 1).

\section{Methods}

GaN-based LEDs with different quantum barriers were grown using metal-organic chemical vapor deposition (MOCVD), and the structure diagrams of these three representative LEDs are shown in Fig. 1. During the growth, trimethygallium (TMGa), trimethylindium (TMIn), silane, $\mathrm{Cp}_{2} \mathrm{Mg}$, and ammonia were used as sources for $\mathrm{Ga}, \mathrm{In}, \mathrm{Si}, \mathrm{Mg}$ and $\mathrm{N}$, respectively. Eight pairs of InGaN MQWs with different indium concentrations in the quantum barriers were deposited on a $120 \mathrm{~nm}$ thick Si-doped n-GaN layer. During the quantum well growth, the temperature and TMIn flow for these three kinds of LEDs were $740{ }^{\circ} \mathrm{C}$ and $160 \mathrm{sccm}$, respectively. While, for different InGaN quantum barriers, the growth temperature were kept at the same $800{ }^{\circ} \mathrm{C}$ but with different TMIn flows at 0,32 , and $160 \mathrm{sccm}$, respectively. Meantime, in order to screen the piezoelectric field, all of the barriers were Si-doped [18]. Finally, $100 \mathrm{~nm}$ thick Mg-doped p-GaN layers were deposited. Details of the growth condition can be found in the previous articles [19-21].

Figure 2 (a) shows the $\omega-2 \theta$-scans of the samples measured by high resolution X-ray diffraction (HRXRD). The main peak of MQWs is labeled as "0th", and its satellite 


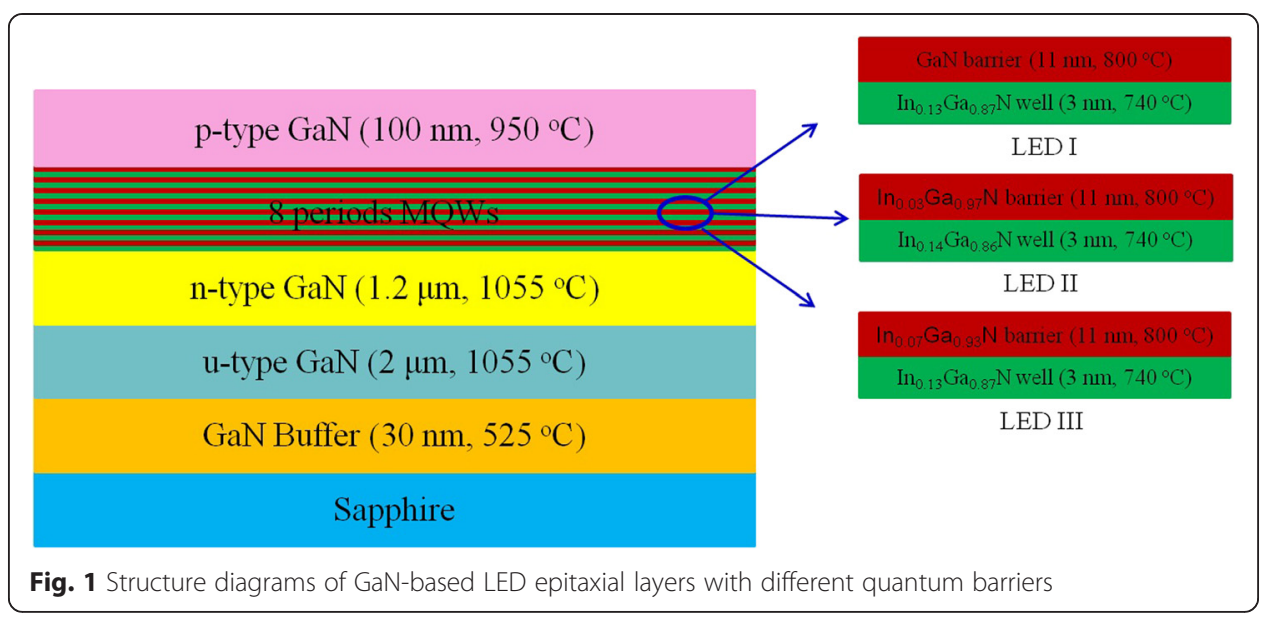

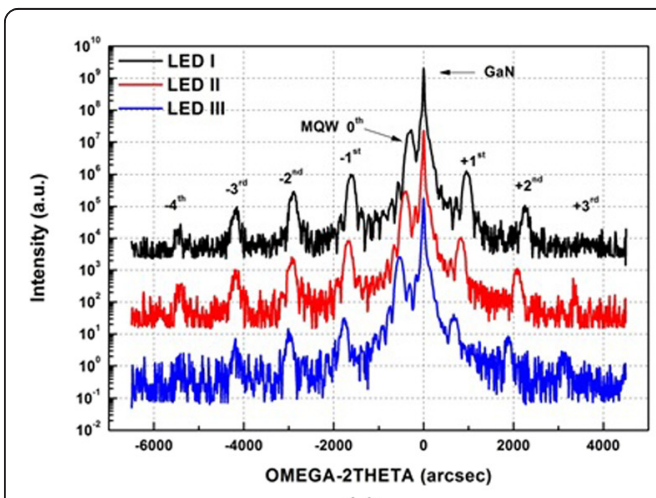

(a)

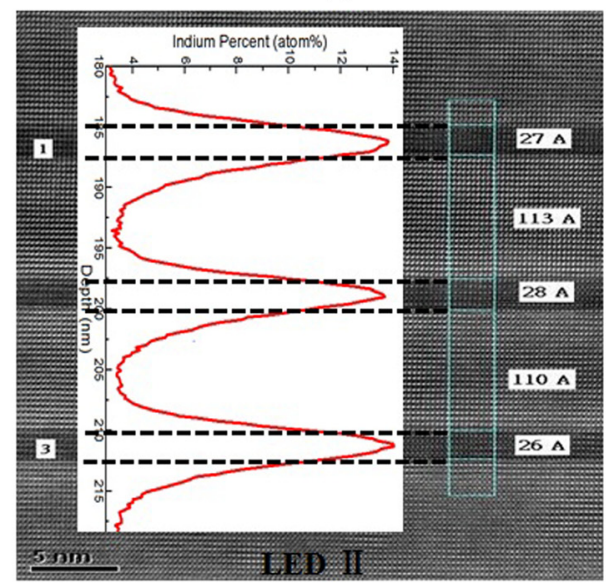

(c)

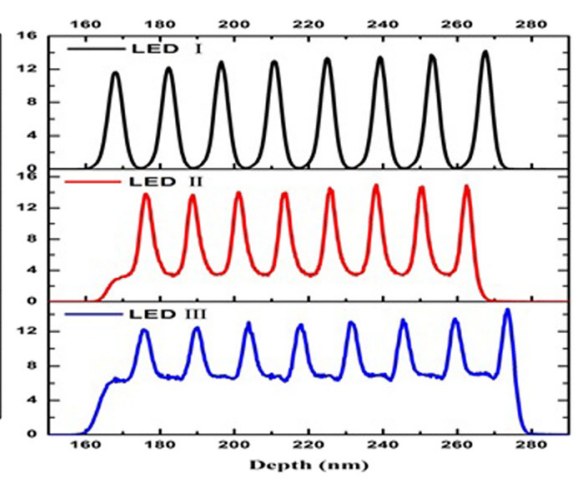

(b)

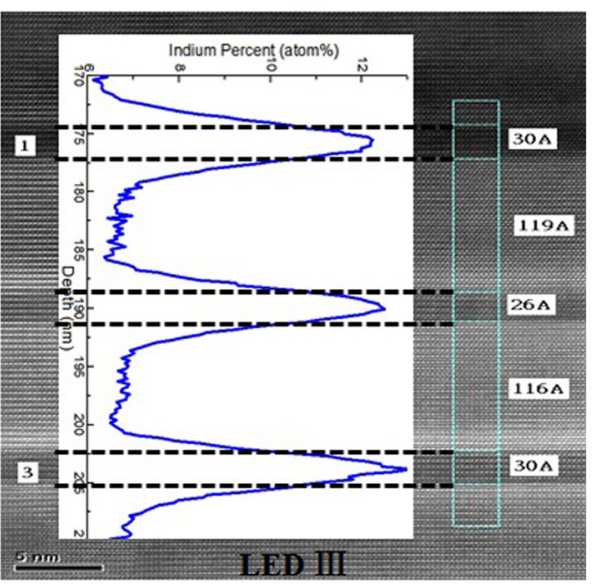

(d)

Fig. 2 a $\omega-2 \theta$ scan curves of these three GaN-based LEDs measured by the high resolution X-ray diffraction; b Indium concentrations measured by SIMS for the MQWs of these three LEDs; $\mathbf{c}$, $\mathbf{d}$ Bright field TEM images and Indium concentrations measured by SIMS for the three pairs of quantum wells/barriers near the $p$-GaN of

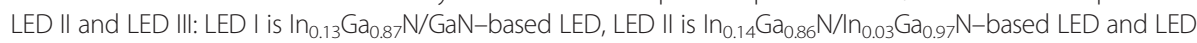
III is $\ln _{0.13} \mathrm{Ga}_{0.87} \mathrm{~N} / \mathrm{In}_{0.07} \mathrm{Ga}_{0.93} \mathrm{~N}$-based LED 
peaks are labeled with numbers. It can be seen that with increasing the TMIn flow, the main (0th) peak of MQWs shifted to lower angle and all of the LEDs show distinctive satellite peaks up to the $4^{\text {th }}$ order, which indicates a comparable crystal quality and abrupt interface of the MQWs [22, 23]. In addition, the full width at half maximum of the (002) rocking curve are 277.6, 244.6 and 279.6 arcsec for LED I, LED II and LED III, respectively, indicating that LED II has a better total crystal quality than the LED I and LED III. The reason may be due to the reduced lattice mismatch and misfit dislocation between the MQWs, but with the indium concentration further increasing, the barrier crystal quality becomes worse due to the indium incorporation [24].

The thicknesses of MQWs and the distributions of indium concentration in the MQW were measured using the bright field transmission electron microscope (BF-TEM) and the secondary ion mass spectroscopy (SIMS). For all the studied LEDs, the thickness and the indium concentrations in the quantum wells are about $3 \mathrm{~nm}$ and $13 \%$, while for the quantum barriers, the thickness is roughly about $11 \mathrm{~nm}$ and the indium concentrations are about 3 and $7 \%$ for LED II and III respectively, and for LED I, the GaN layers were deposited as the quantum barriers, as shown in Fig. 2 (b), (c) and (d). In these three LED structures, in order to more clearly distinguish the original electron distribution in the quantum well region, we did not use the electron blocking layer to eliminate the influence of EBL on electron leakage and polarization field. After the growth, the LED wafers were processed into the device with a rectangular geometry of $0.25 \mathrm{~mm} \times 0.58 \mathrm{~mm}$.

\section{Results and discussion}

Figure 3 (a) shows the current density-voltage characteristics for these three GaN-based LEDs. Compared with LED I, with the indium concentration increasing in the quantum barriers, LED II shows a better electrical performance. However, with the indium further increasing, at the low current region, maybe because of the increased tunneling current due to the indium incorporation, the current density-voltage curve of LED III shifts to smaller current density at the same forward voltage comparing to the LED II [25]. Figure 3 (b) shows the electroluminescence spectra measured at $13.8 \mathrm{~A} / \mathrm{cm}^{2}$ and room temperature for these samples. With the indium increasing in the quantum barriers to $3 \%$, the EL intensity increased by $2.4 \%$ with a blue shift $80 \mathrm{meV}$. However, with the indium further increased to $7 \%$, the EL intensity reduced dramatically with a blue shift $\sim 40 \mathrm{meV}$, which indicates the decrease of polarization field $[17,26]$. Current dependent electroluminescence have also been measured. The inset shows the EL peak position as a function of the current density. For LED I, there is an obvious blue shift about $28 \mathrm{meV}$ with the current increasing. However, for LED II and LED III, there is almost no blue shift at the low current. Afterwards, the peak shift to low energy because of the self-heating effect. This indicates that the polarization field in the LED II and III are negligible.

The energy bandgap of $\operatorname{In}_{\mathrm{x}} \mathrm{Ga}_{1-\mathrm{x}} \mathrm{N}$ over $0 \leq \mathrm{x} \leq 1$ can be expressed by the following empirical expression:

$$
E_{g}\left(\operatorname{In}_{x} G a_{1-x} N\right)=(1-x) E_{g}^{G a N}+x E_{g}^{I n N}-b x(1-x),
$$

where $E_{g}^{G a N}=3.43 \mathrm{eV}, E_{g}^{I n N}=0.71 \mathrm{eV}$ and $b=1.43 \mathrm{eV}$ are the bandgap of GaN and $\mathrm{InN}$, and the bowing parameter [27]. Using the Eq. (3) and band offset ratio of $\Delta E_{C}$ : $\Delta E_{V}=68: 32$ [28], we can calculate the theoretical value of conduction band offset 

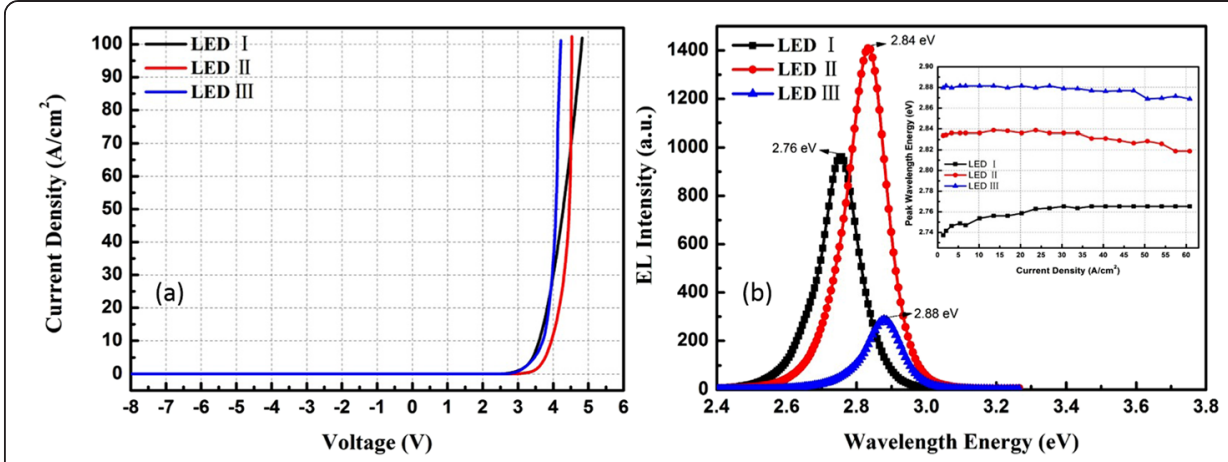

Fig. 3 a The current density-voltage characteristics for these samples and $\mathbf{b}$ the electroluminescence spectra measured at $13.8 \mathrm{~A} / \mathrm{cm}^{2}$ and room temperature for GaN-based LEDs with different quantum barriers: LED I is $\mathrm{In}_{0.13} \mathrm{Ga}_{0.87} \mathrm{~N} / \mathrm{GaN}$-based LED, LED II is $\mathrm{In}_{0.14} \mathrm{Ga}_{0.86} \mathrm{~N} / \mathrm{In}_{0.03} \mathrm{Ga}_{0.97} \mathrm{~N}$-based LED and LED III is $\mathrm{In}_{0.13} \mathrm{Ga}_{0.87} \mathrm{~N} /$ $\mathrm{In}_{0.07} \mathrm{Ga}_{0.93} \mathrm{~N}$-based LED. The inset shows the peak wavelength energy as a function of the current density

$\Delta E_{C}: \Delta E_{C_{-}}$LED I $=0.344 \mathrm{eV}, \Delta E_{C_{-}}$LED II $=0.274 \mathrm{eV}$ and $\Delta E_{C_{-}}$LED III $=0.158 \mathrm{eV}$. An increase of indium concentration in the barrier layers will lead to a small bandgap discontinuity at the well/barrier interfaces and the carrier confinement effect will be reduced, accordingly. But it seems not the only factor which will influence the optical properties of LEDs. Figure 4 shows the dependence of the light-output power (LOP) on the current density for these three samples using the line-symbol curves. Compared with the LED I and LED II, the light output is significantly smaller for LED III. Since the total crystal quality of LED III measured by HRXRD is about the same as the LED I, the reason may be mainly related to the band structure. The lowest band offset $\Delta E_{C}$ and carrier confinement of LED III will lead to a strong electron overflow. While for LED I and LED II, below the current density $13.5 \mathrm{~A} / \mathrm{cm}^{2}$, the light output of LED I with GaN barrier is larger than LED II with $3 \%$ indium composition in the quantum barriers, while with the current density further increasing, the light output for LED II becomes larger. The low EQE of LED II at the low current density may be related to the increased SRH recombination with the indium concentration increasing in the quantum barriers of the LED II than LED I. This trend will be more obvious if we normalized the light intensity to LED I, as illustrated by the line curves in Fig. 4.

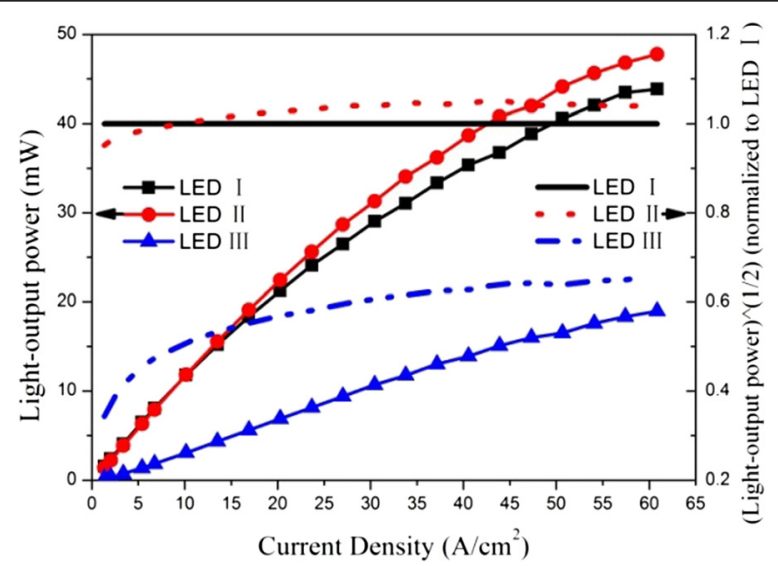

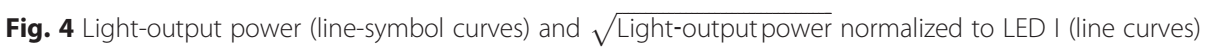
as a function of current density for three LEDs: LED I is $\ln _{0.13} \mathrm{Ga}_{0.87} \mathrm{~N} / \mathrm{GaN}$-based LED, LED II is $\ln _{0.14} \mathrm{Ga}_{0.86} \mathrm{~N}$ / $\mathrm{In}_{0.03} \mathrm{Ga}_{0.97} \mathrm{~N}$-based LED and LED III is $\ln _{0.13} \mathrm{Ga}_{0.87} \mathrm{~N} / \mathrm{In}_{0.07} \mathrm{Ga}_{0.93} \mathrm{~N}$-based LED 
According to the $\mathrm{ABC}$ model, light-output power is proportional to $\mathrm{Bn}^{2}$ (or $\sqrt{\text { Light-outputpower }} \propto \mathrm{n}$ ). So the line curves of $\sqrt{\text { Light-output power }}$ normalized to LED I versus current density also represent the total effective electron concentration which can recombine with the injected holes in the MQWs of these three samples compared with the LED I. With the current density increasing, the $\mathrm{n}$ ratios of LED II and LED III versus LED I increase and saturate at fixed values at 1.04 and 0.64 , respectively. The highest total effective electron concentration in the LED II results in the largest light-output power at the high current density, which indicates that using appropriate indium concentration in the quantum barriers, the reduced carrier confinement will decrease the electron concentration restricted in the quantum wells near the $\mathrm{n}-\mathrm{GaN}$ layer, and increase the total effective electron concentration in the MQWs.

Figure 5 shows the external quantum efficiencies as function of the current density for the LEDs. It can be seen that when the EQEs reach the peak efficiency, at which droop started to occur, the current densities move to high value, from $5.4 \mathrm{~A} / \mathrm{cm}^{2}, 6.8$ $\mathrm{A} / \mathrm{cm}^{2}$ to $30.4 \mathrm{~A} / \mathrm{cm}^{2}$ for LED I, II and III, respectively. The reason may be related to the decreased bandgap discontinuity. With increasing the indium concentration in the quantum barriers, the resulted less carrier confinement will not only reduce the electron concentration in the quantum wells close the n-type region but increase the carrier transport between the quantum wells, which will allow the overall MQWs to tolerant more carriers before the Auger recombination start to take effect and dominate. Accordingly the peak efficiency will move to the high current density, compared with GaN quantum barriers. But with the indium composition further increasing, as for LED III, the non-radiative recombination will be increased because of a strong electron overflow, and as a result the efficiency remains at a low light output level. In addition, compared with the peak EQE of LED I, LED II and LED III, the degree of efficiency droop at current density $61 \mathrm{~A} / \mathrm{cm}^{2}$ are $41 \%, 34 \%$ and $11 \%$, respectively. In general, more accumulated carrier concentration in the individual quantum well will lead to a large Auger recombination. For LED I with the GaN barriers, most of the electron may be confined in the quantum wells near the $\mathrm{n}-\mathrm{GaN}$, in this case, the Auger recombination would be serious. While for LED II with reduced quantum barrier, not only the

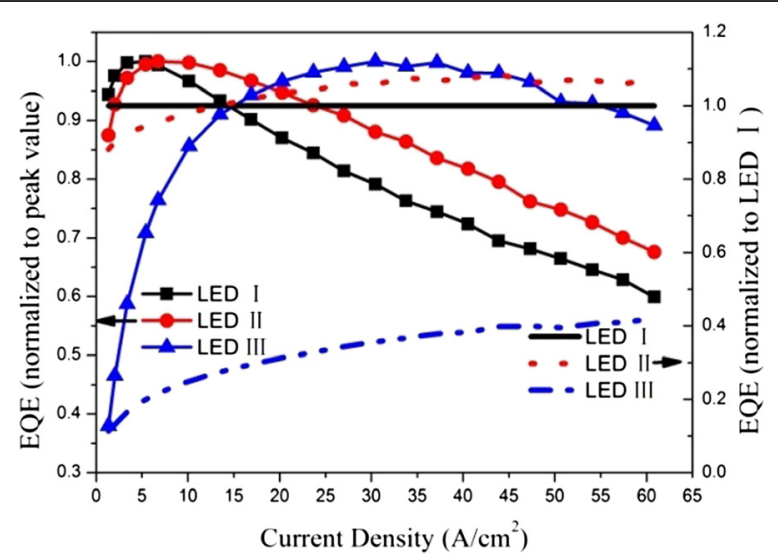

Fig. 5 EQE normalized to peak value (Line-symbol curves) and EQE normalized to LED I (Line curves) as a

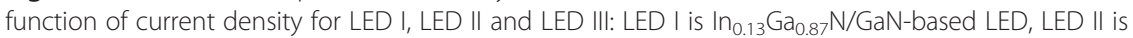
I $\mathrm{n}_{0.14} \mathrm{Ga}_{0.86} \mathrm{~N} / \mathrm{In} \mathrm{n}_{0.03} \mathrm{Ga}_{0.97} \mathrm{~N}$-based LED and LED III is $1 \mathrm{n}_{0.13} \mathrm{Ga}_{0.87} \mathrm{~N} / \mathrm{In} \mathrm{n}_{0.07} \mathrm{Ga}_{0.93} \mathrm{~N}$-based LED 
total electron concentration has been increased, as shown in the Fig. 4, but the droop effect has been improved, the reason may be related to the decreased electron concentration in the individual quantum well close to the $n$-type layer. The less confinement will increase the carrier transport between the wells, which will lead to the less electron accumulation in the single quantum wells. With the indium concentration further increasing, because of the serious electron overflow, the electron concentration in the single well will be seriously reduced, for which there is no droop effect occurred but the EQE has also been restricted.

The EQEs of LED I, LED II and LED III normalized to the LED I have also been calculated, as shown by the line curves in the Fig. 5. With the current density increasing, the normalized EQEs of LED II and LED III increase and saturate at fixed values of 1.07 and 0.41 , respectively, which are just approximately equal to the square of the $n$ ratios of LED II and LED III versus LED I, as calculated in the Fig. 4, indicating that the total effective electron concentration is the key factor, which can also effectively present the EQE of LEDs. As for LED II, it has the highest total effective electron concentration, which means the highest EQE. But at the same time, because of the less electron concentration accumulated in the individual quantum well, this will avoid the Auger recombination and minimize the droop effects at the high current density injection. For LED I, the total effective electron concentration is less than LED II, but because of that the electrons are mostly restricted in the first quantum well close to the n-type $\mathrm{GaN}$ layer, which can lead to a stronger Auger recombination and result in the droop effect.

From above, it can be seen that with the indium composition increasing in the quantum barriers, the bandgap discontinuity will be decreased, which will increase the carrier transport between quantum wells and tolerant more carrier concentrations, therefore the peak efficiency shift to high current. With the current density increasing, if the electron concentration in the single quantum well is high, the Auger recombination would be resulted, which would lead to the droop effect, so in this case, it indicates that electron distribution may be one of key factors, which may both influence the peak efficiency and droop effects. With appropriate indium composition in the quantum barriers, the droop effect of GaNbased LEDs can also be improved, and the reason may be due to more uniformed electron distribution and high effective electron concentration.

\section{Simulations}

In order to further clarify the electron distribution, EQE and droop effect in these LEDs with different quantum barriers, numerical simulations were also performed using the APSYS by Crosslight Software. The simulated structures, such as layer thicknesses, Mg, $\mathrm{Si}$ and In composition concentrations, were set the same as the actual samples and the corresponding nitride physical parameters were used in the calculations. The electron and hole mobility were $100 \mathrm{~cm}^{2} \mathrm{~V}^{-1} \mathrm{~s}^{-1}$ and $7 \mathrm{~cm}^{2} \mathrm{~V}^{-1} \mathrm{~s}^{-1}$, andAuger recombination coefficient for electrons and holes were set as $1 \mathrm{E}-29 \mathrm{~cm}^{6} \mathrm{~s}^{-1}$ and $1 \mathrm{E}-30 \mathrm{~cm}^{6} \mathrm{~s}^{-1}$, respectively. The screening effect factor was 0.5 . The other parameter details can be found in the previous article [29].

Figure 6 (a) and (b) shows the simulation results of the energy band diagram and electric field distribution of LED I, LED II and LED III under the same current density 

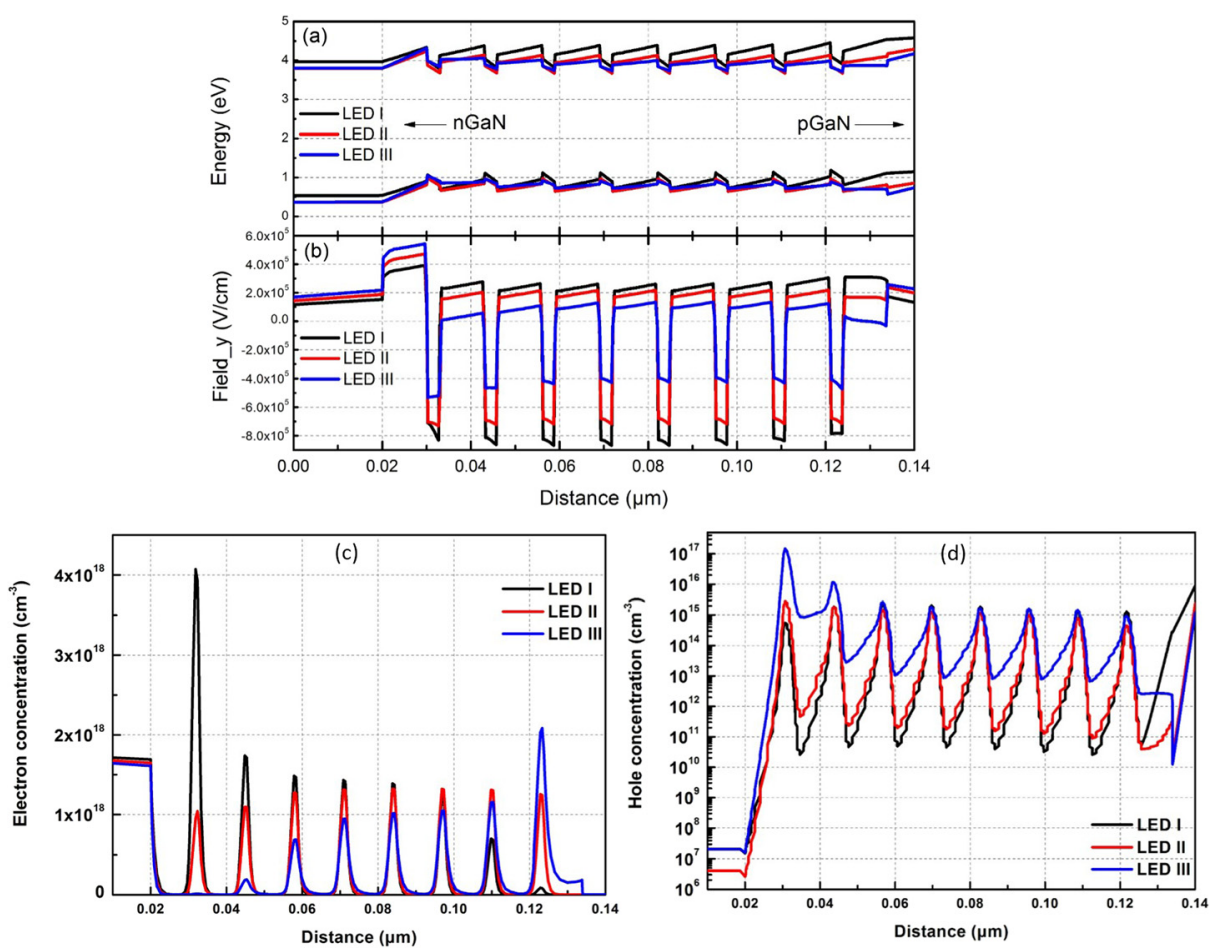

Fig. 6 Simulated a energy band diagram. $\mathbf{b}$ electrical field distribution, c electron concentration and d hole concentration distribution in the MQWs under $30 \mathrm{~A} / \mathrm{cm}^{2}$ for LED I, LED II, and LED III: LED I is $\mathrm{In}_{0.13} \mathrm{Ga}_{0.87} \mathrm{~N} / \mathrm{G}$ aN-based LED, LED II is $\ln _{0.14} \mathrm{Ga}_{0.86} \mathrm{~N} / \mathrm{In}_{0.03} \mathrm{Ga}_{0.97} \mathrm{~N}$-based LED and LED III is $\ln _{0.13} \mathrm{Ga}_{0.87} \mathrm{~N} /$ $\ln _{0.07} \mathrm{Ga}_{0.93} \mathrm{~N}$-based LED

$\left(30 \mathrm{~A} / \mathrm{cm}^{2}\right)$. The indium compositions in the quantum barriers not only reduce the potential barrier of the quantum barriers, but decrease the polarization electric field significantly, which is also consistent with the shift of the peak wavelength shown in Fig. 3 (b). Meanwhile, Fig. 6 (c) and (d) show the electron and hole concentration distribution in the MQWs for these three LEDs. The trends of the electron concentration distribution from the first to the eighth quantum well in these three LEDs are not the same, gradually declining in the LED I, relatively stabilizing in the LED II and gently increasing in the LED III. For LED I with GaN barriers, the electron carriers are mostly concentrated in the first quantum well, and the electron concentration in the first quantum well is also much greater than that in the other two LEDs. Different from the LED with EBL layer [17], since there is no electron block layer in the structure, the electrons will transport to the $\mathrm{p}-\mathrm{GaN}$ layer directly instead of accumulating in the last quantum well close to $\mathrm{p}-\mathrm{GaN}$ layer, and accordingly the radiative recombination will also be restricted by the decreased electron and hole injection concentrations. But with indium incorporation in the quantum barriers, the electrons start to move into the quantum wells close to p-GaN layer. For LED II, the electron concentrations are uniform in all the MQWs, while for LED III the electron carriers are more concentrated in the quantum well close to the p-GaN layer. These results further demonstrate that $\mathrm{GaN}$ quantum barriers would have more strongly confinement effect on the electrons than the InGaN barriers. While using InGaN quantum barriers, a uniform electron distribution can be resulted, which will improve the efficiency droop, but with the indium concentration further increasing, bandgap discontinuity will become smaller, more 
electron overflow will be resulted and the electrons will be more concentrated in the quantum wells near the p-side layer. However, except for the hole concentrations in the quantum wells near the $\mathrm{n}$-GaN layer of LED III, the hole concentrations of these three LEDs are roughly about $1 \mathrm{E} 15 \mathrm{~cm}^{-3}$, much smaller than the electron concentrations. The low hole concentrations may be due to the serious electron overflow result from the lack of EBL layer in the structures. The large hole concentration of LED III in the quantum wells near the $\mathrm{n}-\mathrm{GaN}$ may be resulted from the high indium concentration in the quantum barriers, which reduces the potential barrier and confinement effect of quantum wells and finally leads to the hole accumulation in the quantum wells close to the $\mathrm{n}-\mathrm{GaN}$ layer.

The distributions of radiative recombination rates, Shock-Read-Hall (SRH) recombination rates and Auger recombination rates in the MQWs under $30 \mathrm{~A} / \mathrm{cm} 2$ for these three LED samples were calculated as well, as shown in Fig. 7. Although with the indium concentration increasing, the intrinsic radiative recombination rates in MQWs for LED III are higher than the other two kinds of GaN-based LEDs. the Shock-ReadHall (SRH) recombination rates of LED III are a few times larger than the radiative recombination rates in the quantum wells near the $\mathrm{n}-\mathrm{GaN}$ due to the accumulated hole concentration and low electron concentration, as shown in Fig 6 (c) and (d). This also can explain the reason that the LED III has the lowest light-output power of these three LED samples. For LED I, the Auger recombination rates in the quantum wells near the $\mathrm{n}-\mathrm{GaN}$ are the highest. This is also consistent with the electron concentration calculated in Fig. 6 (c), which indicates that high electron concentration in the individual quantum well would lead to a high Auger recombination rate. However, for LED II,

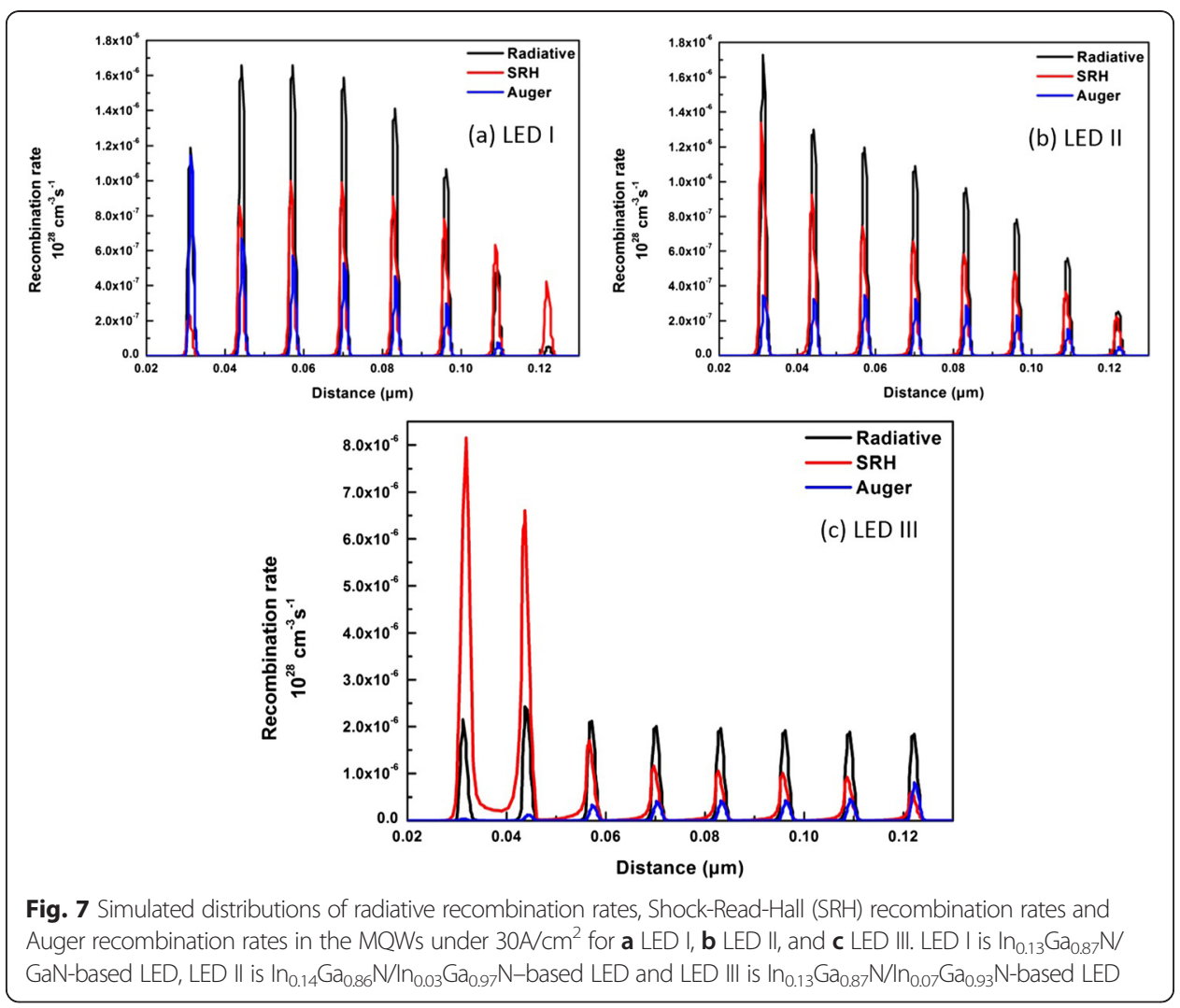


although the radiative recombination rates in the quantum wells are not the highest, the RSH and Auger recombination rates are relatively smaller because of the uniform electron distribution in the MQWs, which lead to the improvement of both EQE and droop effect.

As for LED I, because of GaN barrier layer, the electron concentration in the individual quantum wells of MQWs is larger, and the Auger recombination dominate at the high current density, therefore, it has the biggest droop effect for these three LEDs. While for LED III, because of the serious electron overflow, both the electron concentration in the individual quantum well as well as the total effective carrier concentration in the MQWs are the smallest, so the droop effect can be improved, but the EQE will be influenced. For LED II, electron concentration in the individual quantum well becomes more uniform which will minimize the Auger recombination and improve droop effect. In addition, the less carrier confinement will also allow the MQWs tolerant more total effective carrier concentration and lead to a higher EQE.

\section{Conclusions}

In summary, by changing the indium composition in the barriers of InGaN/GaN MQWs, we investigated the influence of the carrier distribution as well as the corresponding electron overflow and Auger recombination on the efficiency droop. The InGaN quantum barriers with $7 \%$ indium can improve the efficiency droop but decrease the EQE due to the serious electron overflow; the InGaN quantum barriers with $3 \%$ indium can provide a uniform electron distribution, which can not only improve the EQE, but decrease the droop effect compared with the conventional LEDs using the GaN QWs. The results show that the correlation between the electron overflow, electron distribution and Auger recombination. Electron overflow seems mainly influence the peak efficiency, while Auger recombination is the main factor which will determine the efficiency droop and electron distribution will both take an effect on the $\mathrm{EQE}$ and efficiency droop. Using appropriate indium composition in the InGaN barriers can not only improve the electron distribution, but increase the EQE with little droop effect.

Competing interests

The authors declare that they have no competing interest.

\section{Authors' contributions}

JF carried out the experimental study and presented the first draft of the paper. LZ helped to analyze the data, conceive original ideas and draft the paper. NZ provided the samples. JW and $J L$ participated in the technical discussion and helped to draft the manuscript. All authors read and approved the final manuscript.

\section{Acknowledgements}

This work was financially supported by National Natural Science Foundation of China (grants No. 61334009) and China International Science and Technology Cooperation Program (grants No. 2014DFG62280).

Received: 27 March 2015 Accepted: 4 June 2015

Published online: 13 June 2015

References

1. Schubert MF, Xu J, Kim JK, Schubert EF, Kim MH, Yoon S, et al. Polarization-matched GalnN/AIGalnN multi-quantum-well light-emitting diodes with reduced efficiency droop. Appl Phys Lett. 2008;93:041102. doi:10.1063/1.2963029.

2. Wang T, Nakagawa D, Wang J, Sugahara T, Sakai S. Photoluminescence investigation of InGaN/GaN single quantum well and multiple quantum wells. Appl Phys Lett. 1998;73:3571. doi:10.1063/1.122810.

3. Nguyen HP, Cui K, Zhang S, Djavid M, Korinek A, Botton GA, et al. Controlling electron overflow in phosphor-free InGaN/GaN nanowire white light-emitting diodes. Nano Lett. 2012;12:1317-23. doi:10.1021/nl203860b. 
4. Choi S, Ji MH, Kim J, Jin Kim H, Satter MM, Yoder PD, et al. Efficiency droop due to electron spill-over and limited hole injection in III-nitride visible light-emitting diodes employing lattice-matched InAIN electron blocking layers. Appl Phys Lett. 2012;101:161110. doi:10.1063/1.4759044.

5. Monemar B, Sernelius BE. Defect related issues in the "current roll-off" in InGaN based light emitting diodes. Appl Phys Lett. 2007;91:181103. doi:10.1063/1.2801704.

6. Zhao H, Liu G, Arif RA, Tansu N. Current injection efficiency induced efficiency-droop in InGaN quantum well light-emitting diodes. Solid-State Electron. 2010;54:1119-24. doi:10.1016/j.sse.2010.05.019.

7. Iveland J, Martinelli L, Peretti J, Speck JS, Weisbuch C. Direct measurement of auger electrons emitted from a semiconductor light-emitting diode under electrical injection: identification of the dominant mechanism for efficiency droop. Phys Rev Lett. 2013;110:177406. doi:10.1103/PhysRevLett.110.177406.

8. Cho J, Schubert EF, Kim JK. Efficiency droop in light-emitting diodes: challenges and countermeasures. Laser Photon Rev. 2013;7:408-21. doi:10.1002/lpor.201200025.

9. Liu Z, Wei T, Guo E, Yi X, Wang L, Wang J, et al. Efficiency droop in InGaN/GaN multiple-quantum-well blue light-emitting diodes grown on free-standing GaN substrate. Appl Phys Lett. 2011;99:091104. doi:10.1063/1.3605572.

10. Shen YC, Mueller GO, Watanabe S, Gardner NF, Munkholm A, Krames MR. Auger recombination in InGaN measured by photoluminescence. Appl Phys Lett. 2007;91:141101. doi:10.1063/1.2785135.

11. Zhang M, Bhattacharya $P$, Singh J, Hinckley J. Direct measurement of auger recombination in In0.1Ga0.9N/GaN quantum wells and its impact on the efficiency of In0.1 Ga0.9N/GaN multiple quantum well light emitting diodes. Appl Phys Lett. 2009;95:201108. doi:10.1063/1.3266520.

12. Meneghini M, Trevisanello LR, Meneghesso G, Zanoni E. A Review on the Reliability of GaN-Based LEDs. IEEE Trans Device Mater Reliab. 2008;8:323-31. doi:10.1109/TDMR.2008.921527.

13. Dai Q, Shan Q, Cho J, Schubert EF, Crawford MH, Koleske DD, et al. On the symmetry of efficiency-versus-carrierconcentration curves in GalnN/GaN light-emitting diodes and relation to droop-causing mechanisms. Appl Phys Lett. 2011;98:033506. doi:10.1063/1.3544584.

14. Lin GB, Meyaard D, Cho J, Fred Schubert E, Shim H, Sone C. Analytic model for the efficiency droop in semiconductors with asymmetric carrier-transport properties based on drift-induced reduction of injection efficiency. Appl Phys Lett. 2012;100:161106. doi:10.1063/1.4704366.

15. Li H, Li P, Kang J, Li Z, Zhang Y, Li Z, et al. Quantum efficiency enhancement of $530 \mathrm{~nm}$ InGaN green lightemitting diodes with shallow quantum well. Appl Phys Express. 2013;6:052102. doi:10.7567/apex.6.052102.

16. Ryu HY, ShinJ DS, Shim I. Analysis of efficiency droop in nitride light-emitting diodes by the reduced effective volume of InGaN active material. Appl Phys Lett. 2012;100:131109. doi:10.1063/1.3698113.

17. Kuo YK, Chang JY, Tsai MC, Yen H. Advantages of blue InGaN multiple-quantum well light-emitting diodes with InGaN barriers. Appl Phys Lett. 2009;95:011116. doi:10.1063/1.3176406.

18. Ryu MY, Yu PW, Shin EJ, Lee JI, Yu SK, Oh E, et al. Effects of Si-doping in the barriers on the recombination dynamics in $\operatorname{In}_{0.15} \mathrm{Ga}_{0.85} \mathrm{~N} / \mathrm{In}_{0.015} \mathrm{Ga}_{0.985} \mathrm{~N}$ quantum wells. J Appl Phys. 2001;89:634. doi:10.1063/1.1331077.

19. Zhang L, Ding K, Liu NX, Wei TB, Ji XL, Ma P, et al. Theoretical study of polarization-doped GaN-based light-emitting diodes. Appl Phys Lett. 2011;98:101110. doi:10.1063/1.3565173.

20. Liu Z, Ma J, Yi X, Guo E, Wang L, Wang J, et al. p-InGaN/AIGaN electron blocking layer for InGaN/GaN blue light-emitting diodes. Appl Phys Lett. 2012;101:261106. doi:10.1063/1.4773187.

21. Ji X, Wei T, Yang F, Lu H, Wei X, Ma P, et al. Efficiency improvement by polarization-reversed electron blocking structure in GaN-based Light-emitting diodes. Opt Express. 2014;22 Suppl 3:A1001-1008. doi:10.1364/OE.22.0A1001.

22. Anazawa K, Hassanet S, Fujii K, Nakano Y, Sugiyama M. Growth of strain-compensated InGaN/AIN multiple quantum wells on GaN by MOVPE. J Cryst Growth. 2013;370:82-6. doi:10.1016/j.jcrysgro.2012.08.050.

23. Kret S, Dluzewski P, Szczepanska A, Zak M, Czernecki R, Krysko M, et al. Homogenous indium distribution in InGaN/GaN laser active structure grown by LP-MOCVD on bulk GaN crystal revealed by transmission electron microscopy and x-ray diffraction. Nanotechnology. 2007;18:465707. doi:10.1088/0957-4484/18/46/465707.

24. Lin YS, Ma KJ, Hsu C, Feng SW, Cheng YC, Liao CC, et al. Dependence of composition fluctuation on indium content in InGaN/GaN multiple quantum wells. Appl Phys Lett. 2000;77:2988. doi:10.1063/1.1323542.

25. Liu L, Ling M, Yang J, Xiong W, Jia W, Wang G. Efficiency degradation behaviors of current/thermal co-stressed GaN-based blue light emitting diodes with vertical-structure. J Appl Phys. 2012;111:093110. doi:10.1063/1.4712030.

26. Takeuchi T, Wetzel C, Yamaguchi S, Sakai H, Amano H, Akasaki I, et al. Determination of piezoelectric fields in strained GalnN quantum wells using the quantum-confined Stark effect. Appl Phys Lett. 1998;73:1691. doi:10.1063/1.122247

27. Moses PG, Van de Walle CG. Band bowing and band alignment in InGaN alloys. Appl Phys Lett. 2010;96:021908. doi:10.1063/1.3291055.

28. Chen CC, Hsieh KL, Chi GC, Chuo CC, ChyiC Jl, Chang A. Effect of thermal annealing on high indium content InGaN/GaN single quantum well structures. J Appl Phys. 2001;89:5465. doi:10.1063/1.1363678.

29. Saguatti D, Bidinelli L, Verzellesi G, Meneghini M, Meneghesso G, Zanoni E, et al. Investigation of efficiency-droop mechanisms in multi-quantum-well InGaN/GaN blue light-emitting diodes. IEEE Transactions on Electron Devices. 2012:59:1402-9. doi:10.1109/Ted.2012.2186579. 